J. Korean Math. Soc. 52 (2015), No. 6, pp. 1161-1178

http://dx.doi.org/10.4134/JKMS.2015.52.6.1161

\title{
INSERTION-OF-FACTORS-PROPERTY ON SKEW POLYNOMIAL RINGS
}

\author{
Muhittin Başer, Begüm Hiçyilmaz, Fatma Kaynarca, Tai Keun Kwak, \\ AND YANG LEE
}

\begin{abstract}
In this paper, we investigate the insertion-of-factors-property (simply, IFP) on skew polynomial rings, introducing the concept of strongly $\sigma$-IFP for a ring endomorphism $\sigma$. A ring $R$ is said to have strongly $\sigma$-IFP if the skew polynomial ring $R[x ; \sigma]$ has IFP. We examine some characterizations and extensions of strongly $\sigma$-IFP rings in relation with several ring theoretic properties which have important roles in ring theory. We also extend many of related basic results to the wider classes, and so several known results follow as consequences of our results.
\end{abstract}

\section{Introduction}

Throughout this paper, all rings are associative with identity. We denote by $R[x]$ the polynomial ring with an indeterminate $x$ over $R$. $\operatorname{deg} f(x)$ denotes the degree of $f(x) \in R[x]$. Let $\mathbb{Z}$ and $\mathbb{Z}_{n}$ denote the ring of integers and the ring of integers modulo $n$, respectively.

Due to Bell [3], a ring $R$ is called to satisfy the insertion-of-factors-property (simply, an IFP ring) if $a b=0$ implies $a R b=0$ for $a, b \in R$. Narbonne [22] and Shin [26] used the terms semicommutative and SI for the IFP, respectively. Commutative rings have clearly IFP, and any reduced ring (i.e., a ring without nonzero nilpotent elements) has IFP by a simple computation. A ring is called Abelian if every idempotent is central. IFP rings are Abelian by a simple computation.

Another generalization of a reduced ring is an Armendariz ring. Rege and Chhawchharia [25] called a ring $R$ Armendariz if whenever the product of any two polynomials over a ring is zero, then so is the product of any pair of coefficients from the two polynomials. The class of IFP rings and the class of Armendariz rings do not imply each other by [25, Example 3.2] and [13, Example 14]. Note that the polynomial rings over IFP rings need not have IFP by [13, Example 2], but for an Armendariz ring $R, R$ has IFP if and only if

Received October 16, 2014; Revised February 19, 2015.

2010 Mathematics Subject Classification. Primary 16W20, 16U80; Secondary 16 S36.

Key words and phrases. strongly $\sigma$-IFP ring, (strongly) IFP ring, $\sigma$-rigid ring, skew polynomial ring, Dorroh extension, matrix ring. 
$R[x]$ has IFP by [25, Proposition4.6]. Based on this result, Kwak et al. [19] defined a strongly IFP ring: A ring $R$ is called strongly IFP if $R[x]$ has IFP.

For an endomorphism $\sigma$ of a ring $R$, a skew polynomial ring (also called an Ore extension of endomorphism type) $R[x ; \sigma]$ of $R$ is the ring obtained by giving the polynomial ring over $R$ with the new multiplication $x r=\sigma(r) x$ for all $r \in R$. In $R[x ; \sigma]$, consider $1 x^{n}=x^{n}=x 1 x^{n-1}=\sigma(1) x^{n}$ for any $n \geq 1$ where 1 is the identity of $R$. So it is natural to set $\sigma(1)=1$. The set $\left\{x_{j}\right\}_{j \geq 0}$ is easily seen to be a left Ore subset of $R[x ; \sigma]$, so that one can localize $R[x ; \sigma]$ and form the skew Laurent polynomial ring $R\left[x, x^{-1} ; \sigma\right]$. Every element of $R\left[x, x^{-1} ; \sigma\right]$ is a finite sum of elements of the form $x^{-j} r x^{i}$, where $r \in R$ and $i, j$ are nonnegative integers.

According to Krempa [18], an endomorphism $\sigma$ of a $\operatorname{ring} R$ is called rigid if $a \sigma(a)=0$ implies $a=0$ for $a \in R$, and $R$ is called a $\sigma$-rigid ring [8] if there exists a rigid endomorphism $\sigma$ of $R$. Note that any rigid endomorphism of a ring is a monomorphism and $\sigma$-rigid rings are reduced rings by [8, Proposition $5]$, and $R[x ; \sigma]$ is a reduced ring if and only if $R$ is $\sigma$-rigid by [9, Proposition 3] and $R$ is $\sigma$-rigid if and only if $R\left[x, x^{-1} ; \sigma\right]$ is reduced by [23, Theorem 3].

Following Başer et al. [1, Definition 2.1], an endomorphism $\sigma$ of a ring $R$ is called to have IFP if whenever $a b=0$ for $a, b \in R, a R \sigma(b)=0$, and a ring $R$ is called $\sigma$-IFP if there exists an endomorphism $\sigma$ of $R$ having IFP. A ring $R$ is $\sigma$-IFP if and only if for $a, b \in R, a b=0$ implies $a R \sigma^{n}(b)=0$ for all $n \geq 1$ by [1, Remark 2.2]. It is proved that a ring $R$ is $\sigma$-rigid if and only if $R$ is reduced $\sigma$-IFP and $\sigma$ is a monomorphism by [1, Theorem 2.4].

On the other hand, a ring $R$ is called reversible [4] if $a b=0$ implies $b a=0$ for $a, b \in R$. Reversible rings have IFP by simple computation. The reversible ring property cannot go up to polynomial rings by [17, Example 2.1]. Recently, Kaynarca et al. called a ring $R$ strongly $\sigma$-reversible [15, Definition 2.2] if the skew polynomial ring $R[x ; \sigma]$ is reversible, and investigated some characterizations and extensions of strongly $\sigma$-reversible rings. (We change over from "strongly $\sigma$-skew reversible" in [15] to "strongly $\sigma$-reversible", so as to cohere with other related definitions.)

Motivated by the above, in this paper, we extend the insertion-of-factorsproperty of elements and polynomials to one of skew polynomials. We give a generalization of $\sigma$-rigid rings and an extension of strongly IFP rings, introducing the concept of a strongly $\sigma$-IFP ring for an endomorphism $\sigma$ of a ring. Then the structure of strongly $\sigma$-IFP rings and their related properties including extensions are studied and the relations between strongly $\sigma$-IFP rings and generalized reduced rings is also investigated. Consequently, several known results are obtained as corollaries of our results.

From now on, $\sigma$ denotes a nonzero and non identity endomorphism of each given ring, unless specified otherwise. 


\section{Strongly $\sigma$-IFP rings and related properties}

Firstly we start by giving the following example.

Example 2.1. Consider the ring $R=\mathbb{Z}_{2} \oplus \mathbb{Z}_{2}$ with the usual addition and multiplication. Then $R$ has IFP since $R$ is reduced. Let $\sigma: R \rightarrow R$ be defined by $\sigma((a, b))=(b, a)$. For $p(x)=(1,0), q(x)=(0,1) x$ in $R[x ; \sigma], p(x) q(x)=0$ but $p(x) x q(x)=(1,0) x^{2} \neq 0$ for $x \in R[x ; \sigma]$. Thus $R[x ; \sigma]$ does not have IFP.

We can give the following definition.

Definition 2.2. For an endomorphism $\sigma$ of a ring $R$ with $\sigma(1)=1, R$ is called strongly $\sigma$-IFP if $R[x ; \sigma]$ has IFP.

Every strongly $\sigma$-IFP ring has IFP (and so Abelian), and every subring $S$ with $\sigma(S) \subseteq S$ of a strongly $\sigma$-IFP ring is also strongly $\sigma$-IFP. We will freely use these facts without reference. Every domain $D$ with a monomorphism $\sigma$ is clearly strongly $\sigma$-IFP, since $D$ is $\sigma$-rigid. Any strongly $\sigma$-reversible rings are clearly strongly $\sigma$-IFP, but not conversely by the following example.

Example 2.3. Consider the polynomial ring $R=\mathbb{Z}[t]$. Define $\sigma: R \rightarrow R$ by $\sigma(f(t))=f(0)$ where $f(t) \in R$. Then $R$ is not strongly $\sigma$-reversible by $[15$, Example 2.6(2)].

Now, we will show that $R$ is strongly $\sigma$-IFP. Suppose that $p(x) q(x)=0$ for $p(x)=f_{0}(t)+f_{1}(t) x+\cdots+f_{m}(t) x^{m}, q(x)=g_{0}(t)+g_{1}(t) x+\cdots+g_{n}(t) x^{n}$ in $R[x ; \sigma]$, where $f_{i}(t), g_{j}(t) \in R$ for all $i, j$. Without loss of generality, assume that $p(x) \neq 0$.

Case 1. Suppose that $f_{0}(t) \neq 0$. From $f_{0}(t) g_{0}(t)=0$, we have $g_{0}(t)=0$ since $R$ is a domain. From $f_{0}(t) g_{1}(t)+f_{1}(t) \sigma\left(g_{0}(t)\right)=0$ and $g_{0}(t)=0$, we get $g_{1}(t)=0$. Since $f_{0}(t) g_{2}(t)+f_{1}(t) \sigma\left(g_{1}(t)\right)+f_{2}(t) \sigma^{2}\left(g_{0}(t)\right)=0$, we obtain $g_{2}(t)=0$. Continuing this process, we have that $g_{0}(t)=g_{1}(t)=\cdots=g_{n}(t)=$ 0 , i.e., $q(x)=0$. Thus $p(x) R[x ; \sigma] q(x)=0$.

Case 2. Suppose that there is $f_{k}(t) \neq 0$ with $f_{0}(t)=\cdots=f_{k-1}(t)=0$, where $0<k \leq m$. Since $f_{0}(t) g_{k}(t)+f_{1}(t) \sigma\left(g_{k-1}(t)\right)+\cdots+f_{k-1}(t) \sigma^{k-1}\left(g_{1}(t)\right)+$ $f_{k}(t) \sigma^{k}\left(g_{0}(t)\right)=0$, we have $f_{k}(t) \sigma^{k}\left(g_{0}(t)\right)=0$. Since $R$ is a domain and $f_{k}(t) \neq$ $0, \sigma^{k}\left(g_{0}(t)\right)=0$ and so $\sigma\left(g_{0}(t)\right)=0$. From $f_{0}(t) g_{k+1}(t)+f_{1}(t) \sigma\left(g_{k}(t)\right)+\cdots+$ $f_{k}(t) \sigma^{k}\left(g_{1}(t)\right)+f_{k+1}(t) \sigma^{k+1}\left(g_{0}(t)\right)=0$, we have $f_{k}(t) \sigma^{k}\left(g_{1}(t)\right)=0$ and so $\sigma^{k}\left(g_{1}(t)\right)=\sigma\left(g_{1}(t)\right)=0$ by the same argument as above. Continuing this process, we obtain $\sigma\left(g_{j}(t)\right)=0$ for all $0 \leq j \leq n$. This entails $x q(x)=0$, and hence $p(x)\left(r x^{s}\right) q(x)=0$ for any $r \in R$ and $s \geq 0$, noting that $p(x) r q(x)=$ $r p(x) q(x)=0$. This yields $p(x) R[x ; \sigma] q(x)=0$.

By the computations in Cases 1 and 2, we can conclude that $R$ is strongly $\sigma$-IFP.

Note that the endomorphism $\sigma$ of strongly $\sigma$-IFP rings need not be a monomorphism by Example 2.3. 
Let $R$ be a ring with an endomorphism $\sigma$ and $p(x)=\sum_{i=0}^{m} a_{i} x^{i}$ and $q(x)=$ $\sum_{j=0}^{n} b_{j} x^{j}$ in $R[x ; \sigma]$. Recall that a ring $R$ is called $\sigma$-skew Armendariz [9, Definition] if whenever $p(x) q(x)=0, a_{i} \sigma^{i}\left(b_{j}\right)=0$ for all $0 \leq i \leq m$ and $0 \leq$ $j \leq n$, and $R$ is called $\sigma$-skew quasi-Armendariz [10, Definition 2.1] if whenever $p(x) R[x ; \sigma] q(x)=0, a_{i} R \sigma^{i}\left(b_{j}\right)=0$ for all $0 \leq i \leq m$ and $0 \leq j \leq n$. Any $\sigma$ rigid ring is $\sigma$-skew Armendariz by [9, Corollary 4], and the concepts of $\sigma$-skew Armendariz rings and strongly $\sigma$-IFP rings do not imply each other, by help of [13, Example 14] and [25, Example 3.2]. Notice that any $\sigma$-skew Armendariz ring is clearly $\sigma$-skew quasi-Armendariz, when $\sigma$ is an epimorphism.

Proposition 2.4. (1) If $R$ is a strongly $\sigma$-IFP ring, then $R$ has both IFP and $\sigma-I F P$.

(2) Let $R$ be a $\sigma$-skew Armendariz ring and $\sigma$ an epimorphism. Then $R$ has both IFP and $\sigma$-IFP if and only if $R$ is strongly $\sigma$-IFP.

(3) Let $R$ be a strongly $\sigma$-IFP ring and $\sigma$ an epimorphism. Then $R$ is $\sigma$-skew Armendariz if and only if $R$ is $\sigma$-skew quasi-Armendariz.

(4) If $R$ is a strongly $\sigma$-IFP ring, then $\sigma(e)=e$ for any $e^{2}=e \in R$.

Proof. (1) Assume that $R$ is strongly $\sigma$-IFP. Let $a b=0$ for $a, b \in R$. Since $a R[x ; \sigma] b=0$, arb $=0$ and $a s x b=0$ for any $r, s \in R$. Hence $a R b=0$ and $a R \sigma(b)=0$, showing that $R$ has both IFP and $\sigma$-IFP.

(2) It is enough to show the necessity by (1). Assume that $R$ has IFP and $\sigma$ IFP. Let $p(x) q(x)=0$ for $p(x)=\sum_{i=0}^{m} a_{i} x^{i}, q(x)=\sum_{j=0}^{n} b_{j} x^{j} \in R[x ; \sigma]$. Then $a_{i} \sigma^{i}\left(b_{j}\right)=0$ for all $i, j$, since $R$ is $\sigma$-skew Armendariz. Hence $a_{i} R \sigma^{t+i}\left(b_{j}\right)=0$ and so $\left(a_{i} x^{i}\right)\left(R x^{t}\right) b_{j}=0$ for all $i, j$ and $t \geq 0$ by assumption. This implies $p(x)\left(c x^{t}\right) q(x)=0$ for any $c \in R$ and $t \geq 0$. Therefore $p(x) R[x ; \sigma] q(x)=0$, proving that $R$ is strongly $\sigma$-IFP.

(3) Suppose that $R$ is $\sigma$-skew quasi-Armendariz and $p(x) q(x)=0$ for $p(x)=$ $\sum_{i=0}^{m} a_{i} x^{i}$ and $q(x)=\sum_{j=0}^{n} b_{j} x^{j}$ in $R[x ; \sigma]$. Since $R$ is strongly $\sigma$-IFP, $p(x) R[x ; \sigma] q(x)=0$, and so $a_{i} R \sigma^{i}\left(b_{j}\right)=0$ for all $i$ and $j$ by hypothesis. Thus $a_{i} \sigma^{i}\left(b_{j}\right)=0$, completing the proof.

(4) Since $R[x ; \sigma]$ has IFP, it is Abelian. Thus $e x=x e$ for any $e^{2}=e \in R$, and so $\sigma(e)=e$.

Corollary 2.5. (1) If $R$ is a strongly $\sigma$-IFP ring, then $R$ is Abelian.

(2) If $R$ is an Armendariz ring, then $R$ has IFP if and only if $R$ is strongly IFP.

(3) If $R$ is strongly IFP, then $R$ is Armendariz if and only if $R$ is quasiArmendariz (i.e., whenever $f(x) R[x] g(x)=0$ for $f(x)=\sum_{i=0}^{m} a_{i} x^{i}, g(x)=$ $\sum_{j=0}^{n} b_{j} x^{j} \in R[x], a_{i} R b_{j}=0$ for all $\left.i, j[7]\right)$.

Proof. Each proof comes directly from Proposition 2.4(1,2,3).

The following illustrates Proposition 2.4(1,2) in detail. 
Example 2.6. We refer to the argument and ring construction in [16, Example 2.8]. Consider the free algebra $A=\mathbb{Z}_{2}\left\langle a_{0}, a_{1}, a_{2}, b_{0}, b_{1}, b_{2}, c\right\rangle$ with noncommuting indeterminates $a_{0}, a_{1}, a_{2}, b_{0}, b_{1}, b_{2}, c$ over $\mathbb{Z}_{2}$. Define an automorphism $\alpha$ of $A$ by

$$
a_{0}, a_{1}, a_{2}, b_{0}, b_{1}, b_{2}, c \mapsto b_{0}, b_{1}, b_{2}, a_{0}, a_{1}, a_{2}, c,
$$

respectively. Let $B$ be the set of all polynomials with zero constant terms in $A$ and consider the ideal $I$ of $A$ generated by

$$
\begin{aligned}
& a_{0} b_{0}, a_{0} b_{1}+a_{1} b_{0}, a_{0} b_{2}+a_{1} b_{1}+a_{2} b_{0}, a_{1} b_{2}+a_{2} b_{1}, a_{2} b_{2}, a_{0} r b_{0}, a_{2} r b_{2}, \\
& b_{0} a_{0}, b_{0} a_{1}+b_{1} a_{0}, b_{0} a_{2}+b_{1} a_{1}+b_{2} a_{0}, b_{1} a_{2}+b_{2} a_{1}, b_{2} a_{2}, b_{0} r a_{0}, b_{2} r a_{2}, \\
& \left(a_{0}+a_{1}+a_{2}\right) r\left(b_{0}+b_{1}+b_{2}\right),\left(b_{0}+b_{1}+b_{2}\right) r\left(a_{0}+a_{1}+a_{2}\right), \\
& a_{0} a_{0}, a_{2} a_{2}, a_{0} r a_{0}, a_{2} r a_{2}, b_{0} b_{0}, b_{2} b_{2}, b_{0} r b_{0}, b_{2} r b_{2}, r_{1} r_{2} r_{3} r_{4}, \\
& a_{0} a_{1}+a_{1} a_{0}, a_{0} a_{2}+a_{1} a_{1}+a_{2} a_{0}, a_{1} a_{2}+a_{2} a_{1}, \\
& b_{0} b_{1}+b_{1} b_{0}, b_{0} b_{2}+b_{1} b_{1}+b_{2} b_{0}, b_{1} b_{2}+b_{2} b_{1}, \\
& \left(a_{0}+a_{1}+a_{2}\right) r\left(a_{0}+a_{1}+a_{2}\right),\left(b_{0}+b_{1}+b_{2}\right) r\left(b_{0}+b_{1}+b_{2}\right),
\end{aligned}
$$

where $r, r_{1}, r_{2}, r_{3}, r_{4} \in B$. Then clearly $B^{4} \subseteq I$. Set $R=A / I$. Since $\alpha(I) \subseteq I$, we can obtain an automorphism $\sigma$ of $R$ by defining $\sigma(s+I)=\alpha(s)+I$ for $s \in A$. Note that $\sigma^{2}=1_{R}$, where $1_{R}$ denotes the identity endomorphism of $R$. We identify every element of $A$ with its image in $R$ for simplicity. Then $R$ has both IFP and $\sigma$-IFP by [16, Example 2.8].

However, $R$ is neither strongly $\sigma$-IFP nor $\sigma$-skew Armendariz. For $a_{0}+a_{1} x^{2}+$ $a_{2} x^{4}, b_{0}+b_{1} x^{2}+b_{2} x^{4} \in R[x ; \sigma]$, we have $\left(a_{0}+a_{1} x^{2}+a_{2} x^{4}\right)\left(b_{0}+b_{1} x^{2}+b_{2} x^{4}\right)=0$. But

$$
\begin{aligned}
& \left(a_{0}+a_{1} x^{2}+a_{2} x^{4}\right) c x\left(b_{0}+b_{1} x^{2}+b_{2} x^{4}\right) \\
= & \left(a_{0}+a_{1} x^{2}+a_{2} x^{4}\right) c\left(\alpha\left(b_{0}\right)+\alpha\left(b_{1}\right) x^{2}+\alpha\left(b_{2}\right) x^{4}\right) x \\
= & \left(a_{0}+a_{1} x^{2}+a_{2} x^{4}\right) c\left(a_{0}+a_{1} x^{2}+a_{2} x^{4}\right) x \neq 0
\end{aligned}
$$

since $a_{0} c a_{1}+a_{1} c a_{0} \neq 0$, showing that $R[x ; \sigma]$ does not have IFP. Furthermore, we obtain $a_{0} b_{1} \neq 0$ from $\left(a_{0}+a_{1} x^{2}+a_{2} x^{4}\right)\left(b_{0}+b_{1} x^{2}+b_{2} x^{4}\right)=0$, entailing that $R$ is not $\sigma$-skew Armendariz.

Let $\sigma_{\gamma}$ be an endomorphism of a ring $R_{\gamma}$ for each $\gamma \in \Gamma$. Then the map $\sigma: \prod_{\gamma \in \Gamma} R_{\gamma} \rightarrow \prod_{\gamma \in \Gamma} R_{\gamma}$ defined by $\sigma\left(\left(a_{\gamma}\right)_{\gamma}\right)=\left(\sigma_{\gamma}\left(a_{\gamma}\right)\right)_{\gamma}$ for $\left(a_{\gamma}\right)_{\gamma} \in \prod_{\gamma \in \Gamma} R_{\gamma}$ is an endomorphism of the product $\prod_{\gamma \in \Gamma} R_{\gamma}$.

A ring $R$ is called local if $R / J(R)$ is a division ring where $J(R)$ denotes the Jacobson radical of $R, R$ is called semilocal if $R / J(R)$ is semisimple Artinian, and $R$ is called semiperfect if $R$ is semilocal and idempotents can be lifted modulo $J(R)$. Local rings are Abelian and semilocal.

Proposition 2.7. (1) Let $R_{\gamma}$ be a ring with an endomorphism $\sigma_{\gamma}$ for each $\gamma \in \Gamma$. The following are equivalent:

(i) $R_{\gamma}$ is strongly $\sigma_{\gamma}$-IFP for each $\gamma \in \Gamma$. 
(ii) The direct product $\prod_{\gamma \in \Gamma} R_{\gamma}$ of $R_{\gamma},(\gamma \in \Gamma)$ is strongly $\sigma$-IFP.

(iii) The direct sum of $R_{\gamma},(\gamma \in \Gamma)$ is strongly $\sigma-I F P$.

(2) Let $\sigma$ be an endomorphism of a ring $R$ with $\sigma(e)=e$ for any $e^{2}=e \in R$. Then we have the following.

(i) $R$ is strongly $\sigma$-IFP and semiperfect if and only if $R$ is a finite direct sum of local strongly $\sigma_{\gamma}$-IFP rings for $\gamma \in \Gamma$.

(ii) For a central idempotent $e$ of $R, e R$ and $(1-e) R$ are strongly $\sigma-I F P$ if and only if $R$ is strongly $\sigma-I F P$.

Proof. (1) It is enough to show (i) $\Rightarrow$ (ii). Assume (i) and let $p(x) q(x)=0$ where $p(x)=\left(p_{\gamma}(x)\right)_{\gamma}, q(x)=\left(q_{\gamma}(x)\right)_{\gamma} \in \prod_{\gamma \in \Gamma} R_{\gamma}[x ; \sigma]$. Then $p_{\gamma}(x) q_{\gamma}(x)=$ 0 for each $\gamma \in \Gamma$, where $p_{\gamma}(x), q_{\gamma}(x) \in R_{\gamma}\left[x ; \sigma_{\gamma}\right]$. By hypothesis, we have $p_{\gamma}(x)\left(r_{\gamma} x^{s}\right) q_{\gamma}(x)=0$ for every $r_{\gamma} \in R_{\gamma}$, any $s \geq 0$ and each $\gamma \in \Gamma$ and this implies that $p(x)\left(r x^{s}\right) q(x)=0$ for every $r=\left(r_{\gamma}\right)_{\gamma} \in \prod_{\gamma \in \Gamma} R_{\gamma}$ and $s \geq 0$. Therefore the direct product of $R_{\gamma}$ is strongly $\sigma$-IFP.

(2)-(i) Suppose that $R$ is strongly $\sigma$-IFP and semiperfect. Since $R$ is semiperfect, $R$ has a finite orthogonal set $\left\{e_{1}, e_{2}, \ldots, e_{n}\right\}$ of local idempotents whose sum is 1 by [20, Proposition 3.7.2], say $R=\sum_{i=1}^{n} e_{i} R$ such that each $e_{i} R e_{i}$ is a local ring. Since $R$ is Abelian by Corollary 2.5(1). Thus each $e_{i} R$ is an ideal of $R$ with $e_{i} R=e_{i} R e_{i}$. But each $e_{i} R$ is also a strongly $\sigma$-IFP ring as a subring since $\sigma\left(e_{i} R\right) \subseteq e_{i} R$ for each $i$ by assumption.

Conversely assume that $R$ is a finite direct sum of strongly $\sigma_{\gamma}$-IFP local rings. Then $R$ is semiperfect since local rings are semiperfect, and moreover $R$ is strongly $\sigma$-IFP by (1).

(2)-(ii) It comes from (1) with $R \cong e R \oplus(1-e) R$, since the class of strongly $\sigma$-IFP is closed under subrings.

Let $R$ be a ring and $\sigma$ be a monomorphism of $R$. Now we consider the Jordan's construction of an over-ring of $R$ by $\sigma$ (see [14] for more details). Let $A(R, \sigma)$ be the subset $\left\{x^{-i} r x^{i} \mid r \in R\right.$ and $\left.i \geq 0\right\}$ of the skew Laurent polynomial ring $R\left[x, x^{-1} ; \sigma\right]$. Note that for $j \geq 0, x^{j} r=\sigma^{j}(r) x^{j}$ implies $r x^{-j}=x^{-j} \sigma^{j}(r)$ for $r \in R$. This yields that for each $j \geq 0$ we have $x^{-i} r x^{i}=$ $x^{-(i+j)} \sigma^{j}(r) x^{i+j}$. It follows that $A(R, \sigma)$ forms a subring of $R\left[x, x^{-1} ; \sigma\right]$ with the following natural operations: $x^{-i} r x^{i}+x^{-j} s x^{j}=x^{-(i+j)}\left(\sigma^{j}(r)+\sigma^{i}(s)\right) x^{i+j}$ and $\left(x^{-i} r x^{i}\right)\left(x^{-j} s x^{j}\right)=x^{-(i+j)} \sigma^{j}(r) \sigma^{i}(s) x^{i+j}$ for $r, s \in R$ and $i, j \geq 0$. Note that $A(R, \sigma)$ is an over-ring of $R$, and $\sigma$ is actually an automorphism of $A(R, \sigma)$ with $\sigma\left(x^{-i} r x^{i}\right)=x^{-i} \sigma(r) x^{i}$. Jordan showed, with the use of left localization of the skew polynomial $R[x ; \sigma]$ with respect to the set of powers of $x$, that for any pair $(R, \sigma)$, such an extension $A(R, \sigma)$ always exists in [14]. This ring $A(R, \sigma)$ is usually said to be Jordan extension of $R$ by $\sigma$.

Proposition 2.8. Let $R$ be a ring and $\sigma$ a monomorphism of $R$. Then $R$ is a strongly $\sigma$-IFP ring if and only if so is $A(R, \sigma)$.

Proof. It is enough to show the necessity. Let $A=A(R, \sigma)=\left\{t^{-i} r t^{i} \mid r \in R\right.$ and $i \geq 0\}$, using $t$ in place of $x$ in the argument above. Suppose that $R$ is 
strongly $\sigma$-IFP and let $p(x) q(x)=0$ for $p(x)=\sum_{i=0}^{m} a_{i} x^{i}, q(x)=\sum_{j=0}^{n} b_{j} x^{j} \in$ $A[x ; \sigma]$, where $a_{i}=t^{-v(i)} a_{i}^{\prime} t^{v(i)}$ and $b_{j}=t^{-u(j)} b_{j}^{\prime} t^{u(j)}$ for $a_{i}^{\prime}, b_{j}^{\prime} \in R$ and $v(i), u(j) \geq 0$. We use the property that $x^{-i} r x^{i}=x^{-(i+j)} \sigma^{j}(r) x^{i+j}$ for each $j \geq 0$ and that $\sigma$ is an automorphism of $A$. Then we can express all $a_{i}, b_{j}$ by

$$
a_{i}=t^{-s} \alpha_{i} t^{s} \text { and } b_{j}=t^{-s} \beta_{j} t^{s}
$$

for some $s \geq 0$ and $\alpha_{i}, \beta_{j} \in R$. This yields that

$$
\begin{aligned}
0 & =\sum_{k=0}^{m+n}\left(\sum_{k=i+j} a_{i} \sigma^{i}\left(b_{j}\right)\right) x^{k}=\sum_{k=0}^{m+n}\left(\sum_{k=i+j} t^{-s} \alpha_{i} t^{s} \sigma^{i}\left(t^{-s} \beta_{j} t^{s}\right)\right) x^{k} \\
& =\sum_{k=0}^{m+n}\left(\sum_{k=i+j} t^{-s} \alpha_{i} t^{s} t^{-s} \sigma^{i}\left(\beta_{j}\right) t^{s}\right) x^{k}=t^{-s}\left(\sum_{k=0}^{m+n}\left(\sum_{k=i+j} \alpha_{i} \sigma^{i}\left(\beta_{j}\right)\right) x^{k}\right) t^{s} .
\end{aligned}
$$

This entails $\sum_{k=0}^{m+n}\left(\sum_{k=i+j} \alpha_{i} \sigma^{i}\left(\beta_{j}\right)\right) x^{k}=0$. Next let

$$
p^{\prime}(x)=\sum_{i=0}^{m} \alpha_{i} x^{i} \text { and } q^{\prime}(x)=\sum_{j=0}^{n} \beta_{j} x^{j} \in R[x ; \sigma] .
$$

Then we get $p^{\prime}(x) q^{\prime}(x)=0$ by the result above, noting that $p(x)=t^{-s} p^{\prime}(x) t^{s}$ and $q(x)=t^{-s} q^{\prime}(x) t^{s}$. Since $R$ is strongly $\sigma$-IFP, $p^{\prime}(x)\left(r x^{l}\right) q^{\prime}(x)=0$ for all $r \in R$ and $l \geq 0$, entailing that

$$
t^{-u} p^{\prime}(x)\left(r x^{l}\right) q^{\prime}(x) t^{u}=0 \text { for any } u \geq 0 .
$$

Let $a=t^{-c} r t^{c} \in A$ with $r \in R$. Here we can let $c=s$, after resetting $c$ or $s$ in the expression of $a_{i}, b_{j}$ if necessary. Now we have

$$
\begin{aligned}
p(x)\left(a x^{l}\right) q(x) & =\left(t^{-s} p^{\prime}(x) t^{s}\right)\left(a x^{l}\right)\left(t^{-s} q^{\prime}(x) t^{s}\right) \\
& =\left(t^{-s} p^{\prime}(x) t^{s}\right)\left(t^{-s} r t^{s} x^{l}\right)\left(t^{-s} q^{\prime}(x) t^{s}\right) \\
& =t^{-s} p^{\prime}(x) r t^{s} t^{-s} x^{l} q^{\prime}(x) t^{s} \\
& =t^{-s} p^{\prime}(x) r x^{l} q^{\prime}(x) t^{s} \\
& =0
\end{aligned}
$$

by the equality $(*)$. This implies that $p(x) A[x ; \sigma] q(x)=0$, and therefore $A$ is strongly $\sigma$-IFP.

Proposition 2.9. Let $\alpha: R \rightarrow S$ be a ring isomorphism. Then $R$ is a strongly $\sigma-I F P$ if and only if $S$ is a strongly $\alpha \sigma \alpha^{-1}$-IFP ring.

Proof. Note that $p(x)=\sum_{i=0}^{m} a_{i} x^{i}, q(x)=\sum_{j=0}^{n} b_{j} x^{j} \in R[x ; \sigma]$ if and only if $p^{\prime}(x)=\sum_{i=0}^{m} a_{i}^{\prime} x^{i}, q^{\prime}(x)=\sum_{j=0}^{n} b_{j}^{\prime} x^{j} \in S\left[x ; \alpha \sigma \alpha^{-1}\right]$, letting $\alpha\left(a_{i}\right)=a_{i}^{\prime}$, $\alpha\left(b_{j}\right)=b_{j}^{\prime} \in S$ for all $i$ and $j$, and also that $r \in R$ if and only if $r^{\prime} \in S$, letting $\alpha(r)=r^{\prime}$, since $\alpha$ is bijective. 
For any $r \in R$ and $s \geq 0$,

$$
\begin{aligned}
& p(x)\left(r x^{s}\right) q(x)=0 \text { in } R[x ; \sigma] \\
\Leftrightarrow & \sum_{k=0}^{m+n}\left(\sum_{i+j=k} a_{i} \sigma^{i}\left(r \sigma^{s}\left(b_{j}\right)\right)\right) x^{k+s}=0 \\
\Leftrightarrow & \sum_{k=0}^{m+n}\left(\sum_{i+j=k} \alpha\left(a_{i} \sigma^{i}\left(r \sigma^{s}\left(b_{j}\right)\right)\right)\right) x^{k+s}=0, \text { since } \alpha \text { is bijective } \\
\Leftrightarrow & \sum_{k=0}^{m+n}\left(\sum_{i+j=k} \alpha\left(a_{i}\right) \alpha\left(\sigma^{i}\left(r \sigma^{s}\left(b_{j}\right)\right)\right)\right) x^{k+s}=0 \\
\Leftrightarrow & \sum_{k=0}^{m+n}\left(\sum_{i+j=k} \alpha\left(a_{i}\right)\left(\alpha \sigma \alpha^{-1}\right)^{i}\left(\alpha(r)\left(\alpha \sigma \alpha^{-1}\right)^{s} \alpha\left(b_{j}\right)\right)\right) x^{k+s}=0, \\
& \operatorname{since}\left(\alpha \sigma \alpha^{-1}\right)^{t}=\alpha \sigma^{t} \alpha^{-1} \text { for any positive integer } t \\
\Leftrightarrow & \sum_{k=0}^{m+n}\left(\sum_{i+j=k} a_{i}^{\prime}\left(\alpha \sigma \alpha^{-1}\right)^{i}\left(r^{\prime}\left(\alpha \sigma \alpha^{-1}\right)^{s}\left(b_{j}^{\prime}\right)\right)\right) x^{k+s}=0 \\
\Leftrightarrow & p^{\prime}(x)\left(r^{\prime} x^{s}\right) q^{\prime}(x)=0 \text { in } S\left[x ; \alpha \sigma \alpha^{-1}\right] \text { for any } r^{\prime} \in S \text { and } s \geq 0 .
\end{aligned}
$$

Theorem 2.10. A ring $R$ is $\sigma$-rigid if and only if $R$ is a semiprime strongly $\sigma-I F P$ ring and $\sigma$ is a monomorphism of $R$.

Proof. If $R$ is $\sigma$-rigid (i.e., $R[x ; \sigma]$ is reduced), then $R$ is clearly strongly $\sigma$-IFP, and moreover $R$ is reduced (so semiprime) and $\sigma$ is a monomorphism by [8, Proposition 5].

Now, suppose that $R$ is semiprime strongly $\sigma$-IFP and $\sigma$ is a monomorphism of $R$. Let $\sigma(a) a=0$ for $a \in R$. Since $R$ is $\sigma$-IFP, $\sigma(a) R \sigma(a)=0$. Then $\sigma(a)=0$ and so $a=0$ by hypothesis. By [12, Proposition 2.4], $R$ is $\sigma$-rigid.

The following example shows that the conditions " $R$ is a semiprime ring" and " $\sigma$ is a monomorphism" in Theorem 2.10 cannot be dropped, respectively.

Example 2.11. (1) Consider a ring

$$
R=\left\{\left(\begin{array}{cc}
a & b \\
0 & a
\end{array}\right) \mid a, b \in \mathbb{Z}\right\}
$$

Let $\sigma: R \rightarrow R$ be an automorphism defined by

$$
\sigma\left(\left(\begin{array}{ll}
a & b \\
0 & a
\end{array}\right)\right)=\left(\begin{array}{rr}
a & -b \\
0 & a
\end{array}\right) .
$$

Clearly, $R$ is not semiprime and so $R$ is not $\sigma$-rigid. By [15, Propostion 2.13], $R$ is strongly $\sigma$-reversible and hence $R$ is strongly $\sigma$-IFP. 
(2) Recall the strongly $\sigma$-IFP $\operatorname{ring} R=\mathbb{Z}[t]$ and the endomorphism $\sigma$ of $R$ which is not a monomorphism, as in Example 2.3. Then $R$ is a domain and so semiprime. But $R$ is not $\sigma$-rigid, since $x \sigma(x)=0$ for $0 \neq x \in R$.

Jordan's construction also yields $R\left[x, x^{-1} ; \sigma\right] \cong A(R, \sigma)\left[x, x^{-1} ; \sigma\right]$, by way of an isomorphism defined by $x^{-i} r x^{j} \mapsto \sigma^{i}(r) x^{j-i}$, noting $\sigma^{i}(r)=\sigma^{i}(r) x^{-u} x^{u}=$ $x^{-u} \sigma^{u+i}(r) x^{u}$ for any $u \geq 0$ and $r \in R$. So Proposition 2.8 provides the following.

Corollary 2.12. Let $R$ be a semiprime ring and $\sigma$ a monomorphism of $R$. Then the following are equivalent:

(1) $R$ is strongly $\sigma$-reversible.

(2) $R$ is strongly $\sigma-I F P$.

(3) $R$ is $\sigma$-IFP.

(4) $R$ is $\sigma$-rigid.

(5) $R\left[x, x^{-1} ; \sigma\right]$ is a reduced ring.

(6) $A(R, \sigma)$ is strongly $\sigma$-reversible.

(7) $A(R, \sigma)$ is strongly $\sigma-I F P$.

(8) $A(R, \sigma)$ is $\sigma$-IFP.

(9) $A(R, \sigma)$ is $\sigma$-rigid.

Proof. $(4) \Rightarrow(1) \Rightarrow(2) \Rightarrow(3)$ and $(9) \Rightarrow(6) \Rightarrow(7) \Rightarrow(8)$ are obvious. $\quad(3) \Rightarrow(4)$ and $(8) \Rightarrow(9)$ follow from the proof of Theorem 2.10 , and $(2) \Leftrightarrow(7)$ is shown by Proposition 2.8. We see that $R$ is $\sigma$-rigid if and only if $R\left[x, x^{-1} ; \sigma\right]$ is reduced, in $\left[23\right.$, Theorem 3]. Since $R\left[x, x^{-1} ; \sigma\right]$ is isomorphic to $A(R, \sigma)\left[x, x^{-1} ; \sigma\right]$, we can obtain the equivalence of (4) and (5).

McCoy [21] showed that if two polynomials annihilate each other over a commutative ring, then each polynomial has a nonzero annihilator in the base ring. Nielsen [24] and Rege-Chhawchharia [25] called a noncommutative ring $R$ right $M c C o y$ (resp., left McCoy) if whenever any nonzero polynomials $f(x), g(x) \in R[x]$ satisfy $f(x) g(x)=0$, then $f(x) c=0$ (resp., $c g(x)=0$ ) for some nonzero $c \in R$, and a ring $R$ is called McCoy if it is both left and right McCoy. Reversible rings are McCoy by [24, Theorem 2], but there exists an IFP ring which is not McCoy [24, Section 3]. The McCoy condition on a ring is also extended on the skew polynomial ring. A ring $R$ is called $\sigma$-skew $M c$ Coy [2, Defiition 1] if for any nonzero polynomials $p(x)$ and $q(x) \in R[x ; \sigma]$, $p(x) q(x)=0$ implies $p(x) c=0$ for some nonzero $c \in R$.

Proposition 2.13. If $R$ is a strongly $\sigma$-IFP ring, then $R$ is a $\sigma$-skew McCoy ring.

Proof. Suppose that $R$ is a strongly $\sigma$-IFP ring. Let $p(x) q(x)=0$ for any nonzero skew polynomials $p(x), q(x) \in R[x ; \sigma]$. Then $p(x) R[x ; \sigma] q(x)=0$. By [11, Theorem 1], there exists $0 \neq c \in R$ such that $p(x) R[x ; \sigma] c=0$ and so $p(x) c=0$, showing that $R$ is $\sigma$-skew McCoy. 
Corollary 2.14 ([19, Proposition 3.19]). If $R$ is a strongly IFP ring, then $R$ is a McCoy ring.

\section{Examples of strongly $\sigma$-IFP rings}

Let $R$ be an algebra over a commutative ring $S$. Following [5], the Dorroh extension of $R$ by $S$ is the Abelian group $D=R \oplus S$ with multiplication given by $\left(r_{1}, s_{1}\right)\left(r_{2}, s_{2}\right)=\left(r_{1} r_{2}+s_{1} r_{2}+s_{2} r_{1}, s_{1} s_{2}\right)$, where $r_{i} \in R$ and $s_{i} \in S$. For an endomorphism $\alpha$ of $R$ and the Dorroh extension $D$ of $R$ by $S, \bar{\alpha}: D \rightarrow D$ defined by $\bar{\alpha}(r, s)=(\alpha(r), s)$ is an $S$-algebra homomorphism.

Theorem 3.1. Let $R$ be an algebra over a commutative domain $S$ and $\sigma$ an endomorphism of $R$. Then $R$ is strongly $\sigma$-IFP if and only if the Dorroh extension $D$ of $R$ by $S$ is strongly $\bar{\sigma}-I F P$.

Proof. Recall that $\sigma(1)=1$ and note that $s \in S$ is identified with $s 1 \in R$ and so $R=\{r+s \mid(r, s) \in D\}$. It is sufficient to show that the Dorroh extension $D$ is strongly $\bar{\sigma}$-IFP when $R$ is strongly $\sigma$-IFP. Assume that $R$ is strongly $\sigma$-IFP. Let $p(x) q(x)=0$ for $p(x)=\sum_{i=0}^{m}\left(a_{i}, b_{i}\right) x^{i}, q(x)=\sum_{j=0}^{n}\left(c_{j}, d_{j}\right) x^{j} \in D[x ; \bar{\sigma}]$. Then we have

(1)

$$
\sum_{k=0}^{m+n}\left(\sum_{i+j=k}\left(a_{i}, b_{i}\right) \bar{\sigma}^{i}\left(c_{j}, d_{j}\right)\right) x^{k}=\sum_{k=0}^{m+n}\left(\sum_{i+j=k}\left(a_{i}, b_{i}\right)\left(\sigma^{i}\left(c_{j}\right), d_{j}\right)\right) x^{k}=0 .
$$

Put $f(x)=\sum_{i=0}^{m} b_{i} x^{i}, g(x)=\sum_{j=0}^{n} d_{j} x^{j} \in S[x]$, then we get $f(x) g(x)=0$ by Eq. (1). Since $S[x]$ is a domain, $f(x)=0$ or $g(x)=0$. Note that $\sigma(s)=s$ for all $s \in S$, since $\sigma(1)=1$. We will freely use this fact without reference in the following procedure.

If $f(x)=0$, then we have $\sum_{k=0}^{m+n}\left(\sum_{i+j=k}\left(a_{i}\left(\sigma^{i}\left(c_{j}\right)+d_{j}\right), 0\right)\right) x^{k}=0$ by Eq. (1), and so we get

(2) $0=\sum_{k=0}^{m+n}\left(\sum_{i+j=k} a_{i}\left(\sigma^{i}\left(c_{j}\right)+d_{j}\right)\right) x^{k}=\sum_{k=0}^{m+n}\left(\sum_{i+j=k} a_{i} \sigma^{i}\left(c_{j}+d_{j}\right)\right) x^{k}$.

We let $p^{\prime}(x)=\sum_{i=0}^{m} a_{i} x^{i}, q^{\prime}(x)=\sum_{j=0}^{n}\left(c_{j}+d_{j}\right) x^{j} \in R[x ; \sigma]$. Then we get $p^{\prime}(x) q^{\prime}(x)=0$ by Eq. (2). Since $R$ is strongly $\sigma$-IFP, $p^{\prime}(x)\left((r+s) x^{t}\right) q^{\prime}(x)=0$ for any $r+s \in R$ and $t \geq 0$, and we obtain

$$
\begin{aligned}
0 & =\sum_{k=0}^{m+n}\left(\sum_{i+j=k} a_{i} \sigma^{i}\left((r+s) \sigma^{t}\left(c_{j}+d_{j}\right)\right)\right) x^{k+t} \\
& =\sum_{k=0}^{m+n}\left(\sum_{i+j=k} a_{i}\left(\sigma^{i}(r)+s\right)\left(\sigma^{i+t}\left(c_{j}\right)+d_{j}\right)\right) x^{k+t} .
\end{aligned}
$$


Hence,

$$
\begin{aligned}
& \sum_{k=0}^{m+n}\left(\sum_{i+j=k}\left(a_{i}, 0\right) \bar{\sigma}^{i}\left((r, s) \bar{\sigma}^{t}\left(\left(c_{j}, d_{j}\right)\right)\right)\right) x^{k+t} \\
= & \sum_{k=0}^{m+n}\left(\sum_{i+j=k}\left(a_{i}, 0\right)\left(\sigma^{i}(r), s\right)\left(\sigma^{i+t}\left(c_{j}\right), d_{j}\right)\right) x^{k+t} \\
= & \sum_{k=0}^{m+n}\left(\sum_{i+j=k}\left(a_{i}\left(\sigma^{i}(r)+s\right)\left(\sigma^{i+t}\left(c_{j}\right)+d_{j}\right), 0\right)\right) x^{k+t}=0,
\end{aligned}
$$

showing that $p(x)(r, s) x^{t} q(x)=0$ for any $(r, s) \in D$ and $t \geq 0$.

In case of $g(x)=0$, we can show that $p(x)(r, s) x^{t} q(x)=0$ for any $(r, s) \in D$ and $t \geq 0$ by the similar argument to above.

Consequently, the Dorroh extension $D$ of $R$ by $S$ is strongly $\bar{\sigma}$-IFP if $R$ is strongly $\sigma$-IFP.

By Proposition 2.7(2-ii) and Theorem 3.1, we have the following.

Corollary 3.2 ([19, Proposition 3.17]). (1) For a central idempotent e of a ring $R, R$ is strongly IFP if and only if $e R$ and $(1-e) R$ are strongly IFP.

(2) Let $R$ be an algebra over a commutative domain $S$, and $D$ be the Dorroh extension of $R$ by $S$. Then $R$ is strongly IFP if and only if $D$ is strongly IFP.

Recall that if $\sigma$ is an endomorphism of a ring $R$, then the map $\bar{\sigma}: R[x] \rightarrow$ $R[x]$ defined by

$$
\bar{\sigma}\left(\sum_{i=0}^{m} a_{i} x^{i}\right)=\sum_{i=0}^{m} \sigma\left(a_{i}\right) x^{i}
$$

is an endomorphism of $R[x]$. This map extends $\sigma$ clearly.

Theorem 3.3. Let $R$ be a ring with an endomorphism $\sigma$ such that $\sigma^{t}=1_{R}$ for some positive integer $t$, where $1_{R}$ denotes the identity endomorphism of $R$. Then $R$ is strongly $\sigma$-IFP if and only if $R[x]$ is strongly $\bar{\sigma}-I F P$.

Proof. We apply the proof of [15, Theorem 3.6]. Assume that $R$ is strongly $\sigma$-IFP, and let $p(y)=f_{0}(x)+f_{1}(x) y+\cdots+f_{m}(x) y^{m}, q(y)=g_{0}(x)+g_{1}(x) y+$ $\cdots+g_{n}(x) y^{n}$ in $R[x][y ; \bar{\sigma}]$ such that $p(y) q(y)=0$. Let

$$
f_{i}(x)=a_{i_{0}}+a_{i_{1}} x+\cdots+a_{i_{w_{i}}} x^{w_{i}} \text { and } g_{j}(x)=b_{j_{0}}+b_{j_{1}} x+\cdots+b_{j_{v_{j}}} x^{v_{j}}
$$

for each $0 \leq i \leq m$, and $0 \leq j \leq n$, where $a_{i_{0}}, \ldots, a_{w_{i}}, b_{j_{0}}, \ldots, b_{v_{j}} \in R$. Set

$$
C=\left\{a_{i_{0}}, \ldots, a_{i_{w_{i}}}, b_{j_{0}}, \ldots, b_{j_{v_{j}}} \mid 0 \leq i \leq m \text { and } 0 \leq j \leq n\right\} .
$$

Let $R[y ; \sigma]$ be the skew polynomial ring with an indeterminate $y$ over $R$, subject to $y r=\sigma(r) y$ for $r \in R$. We can rewrite $p(y)$ and $q(y)$ by $h_{0}(y)+h_{1}(y) x+\cdots+h_{u}(y) x^{u}$ and $l_{0}(y)+l_{1}(y) x+\cdots+l_{s}(y) x^{s} \in R[y ; \sigma][x]$, 
where $h_{i}(y), l_{j}(y) \in R[y ; \sigma]$, and $R[y ; \sigma][x]$ is the polynomial ring with an indeterminate $x$ over $R[y ; \sigma]$. Set $h(x)=p(y)$ and $l(x)=q(y)$, then $h(x) l(x)=0$.

Take a positive integer $k$ such that $k$ is larger than the maximal integer in

$$
\left\{\operatorname{deg} h_{i}(y), \operatorname{deg} l_{j}(y) \mid 0 \leq i \leq u \text { and } 0 \leq j \leq s\right\},
$$

where the degree of zero polynomial is taken to be 0 . Set

$$
\begin{aligned}
h\left(y^{t k}\right) & =h_{0}(y)+h_{1}(y) y^{t k}+\cdots+h_{u}(y) y^{m t k} \text { and } \\
l\left(y^{t k}\right) & =l_{0}(y)+l_{1}(y) y^{t k}+\cdots+l_{s}(y) y^{n t k} .
\end{aligned}
$$

Then $h\left(y^{t k}\right), l\left(y^{t k}\right) \in R[y ; \sigma]$. Note that the set of coefficients of the $h_{i}(y)$ 's (resp., $l_{j}(y)$ 's) equals the set of coefficients of $h\left(y^{t k}\right)$ (resp., $l\left(y^{t k}\right)$ ). Moreover this set is equal to the set $C$. Therefore, since $p(y) q(y)=0=h(x) l(x)$ and $\sigma^{t k}=1_{R}$, we get $h\left(y^{t k}\right) l\left(y^{t k}\right)=0$ in $R[y ; \sigma]$, noting that $\bar{\sigma}(r)=\sigma(r)$ for $r \in R$. Then we have $h\left(y^{t k}\right) r y^{\gamma} l\left(y^{t k}\right)=0$ for any $r \in R$ and $\gamma \geq 0$, since $R$ is strongly $\sigma$-IFP. Then

$$
\sum_{\iota=0}^{m+n}\left(\sum_{i+j=\iota} a_{\lambda_{i}} \sigma^{\lambda_{i}}\left(r \sigma^{\gamma}\left(b_{\epsilon_{j}}\right)\right)\right) y^{\iota+\gamma}=0 \text { for all } \lambda_{i} \text { and } \epsilon_{j} .
$$

Thus $\sum_{i+j=\iota} h_{i}(y)\left(r y^{\gamma}\right) l_{j}(y)=0$ for each $0 \leq \iota \leq m+n$ since $\sigma^{t k}=1_{R}$, entailing $p(y)\left(r y^{\gamma}\right) q(y)=0$ for any $r \in R$ and $\gamma \geq 0$. Therefore $R[x]$ is strongly $\bar{\sigma}$-IFP.

The converse comes from the fact that the class of strongly $\sigma$-IFP rings is closed under subrings, recalling that $\bar{\sigma}(r)=\sigma(r)$ for $r \in R$.

The ring of Laurent polynomials in $x$, coefficients in a ring $R$, consists of all formal sums $\sum_{i=k}^{n} a_{i} x^{i}$ with the usual addition and multiplication, where $a_{i} \in$ $R$ and $k, n$ are (possibly negative) integers. We denote this ring by $R\left[x ; x^{-1}\right]$. The map $\bar{\sigma}: R\left[x ; x^{-1}\right] \rightarrow R\left[x ; x^{-1}\right]$ defined by $\bar{\sigma}\left(\sum_{i=k}^{n} a_{i} x^{i}\right)=\sum_{i=k}^{n} \sigma\left(a_{i}\right) x^{i}$ extends $\sigma$ and is also an endomorphism of $R\left[x ; x^{-1}\right]$.

Proposition 3.4. Let $R$ be a ring with an endomorphism $\sigma$ such that $\sigma^{t}=1_{R}$ for some positive integer $t$. Then $R$ is strongly $\sigma$-IFP if and only if $R\left[x ; x^{-1}\right]$ is strongly $\bar{\sigma}-I F P$.

Proof. Assume that $R$ is strongly $\sigma$-IFP, and let $p(y)=f_{0}(x)+f_{1}(x) y+\cdots+$ $f_{m}(x) y^{m}, q(y)=g_{0}(x)+g_{1}(x) y+\cdots+g_{n}(x) y^{n}$ in $R\left[x ; x^{-1}\right][y ; \bar{\sigma}]$ such that $p(y) q(y)=0$. Let

$$
f_{i}(x)=\sum_{u=s_{i}}^{w_{i}} a_{u} x^{u} \text { and } g_{j}(x)=\sum_{v=k_{j}}^{z_{j}} b_{v} x^{v}
$$

for each $0 \leq i \leq m$, and $0 \leq j \leq n$, where $a_{s_{i}}, \ldots, a_{w_{i}}, b_{k_{j}}, \ldots, b_{z_{j}} \in R$ and $s_{i}, w_{i}, k_{j}, z_{j} \in \mathbb{Z}$. Take positive integers $s$ and $k$ such that $s=\max \left\{\left|s_{i}\right| \mid\right.$ $i=0,1, \ldots, m\}$ and $k=\max \left\{\left|k_{j}\right| \mid j=0,1, \ldots, n\right\}$. Let $p(y)=x^{s} f(y)=$ $f_{0}^{\prime}(x)+f_{1}^{\prime}(x) y+\cdots+f_{m}^{\prime}(x) y^{m}, q(y)=x^{k} g(y)=g_{0}^{\prime}(x)+g_{1}^{\prime}(x) y+\cdots+g_{n}^{\prime}(x) y^{n}$, 
where $f_{i}^{\prime}(x)=f_{i}(x) x^{s}$ and $g_{j}^{\prime}(x)=g_{j}(x) x^{k}$. By the similar computation to the proof of Theorem 3.3 , we can show that $R\left[x ; x^{-1}\right]$ is strongly $\bar{\sigma}$-IFP.

By Theorem 3.3 and Proposition 3.4, we obtain the following result which includes [19, Theorem 3.14].

Corollary 3.5. For a ring $R$, the following are equivalent:

(1) $R$ is strongly IFP.

(2) $R[x]$ is strongly IFP.

(3) $R\left[x ; x^{-1}\right]$ is strongly IFP.

An element $u$ of a ring $R$ is right regular if $u r=0$ implies $r=0$ for $r \in R$. Similarly, left regular is defined, and regular means if it is both left and right regular (and hence not a zero divisor). Assume that $M$ is a multiplicatively closed subset of $R$ consisting of central regular elements. Let $\sigma$ be an automorphism of $R$ and assume $\sigma(m)=m$ for every $m \in M$. Then $\sigma\left(m^{-1}\right)=m^{-1}$ in $M^{-1} R$ and the induced map $\bar{\sigma}: M^{-1} R \rightarrow M^{-1} R$ defined by $\bar{\sigma}\left(u^{-1} a\right)=u^{-1} \sigma(a)$ is also an automorphism.

Proposition 3.6. Let $R$ be a ring with an automorphism $\sigma$ and assume that there exists a multiplicatively closed subset $M$ of $R$ consisting of central regular elements and $\sigma(m)=m$ for every $m \in M$. Then $R$ is a strongly $\sigma$-IFP ring if and only if $M^{-1} R$ is a strongly $\bar{\sigma}-I F P$ ring.

Proof. Let $R$ be a strongly $\sigma$-IFP, and suppose that $P(x) Q(x)=0$ for $P(x)=$ $u^{-1}\left(a_{0}+a_{1} x+\cdots+a_{k} x^{k}\right), Q(x)=v^{-1}\left(b_{0}+b_{1} x+\cdots+b_{n} x^{n}\right) \in M^{-1} R[x ; \bar{\sigma}]$. Then

$$
\begin{aligned}
0 & =P(x) Q(x) \\
& =u^{-1}\left(a_{0} v^{-1}+a_{1} \sigma(v)^{-1} x+\cdots+a_{k} \sigma^{k}(v)^{-1} x^{k}\right)\left(b_{0}+b_{1} x+\cdots+b_{n} x^{n}\right) .
\end{aligned}
$$

Since $\sigma^{i}(v)^{-1}=v^{-1}$ for $0 \leq i \leq k$ by hypothesis, we have

$$
\begin{aligned}
0 & =P(x) Q(x) \\
& =(u v)^{-1}\left(a_{0}+a_{1} x+\cdots+a_{k} x^{k}\right)\left(b_{0}+b_{1} x+\cdots+b_{n} x^{n}\right) .
\end{aligned}
$$

Let $p(x)=a_{0}+a_{1} x+\cdots+a_{k} x^{k}$ and $q(x)=b_{0}+b_{1} x+\cdots+b_{n} x^{n} \in R[x]$. Then we get $p(x) q(x)=0$ and hence $p(x) R[x ; \sigma] q(x)=0$, since $R$ is a strongly $\sigma$-IFP. Then for any $r^{-1} s \in M^{-1} R$ and $t \geq 0$,

$$
\begin{aligned}
& P(x)\left(r^{-1} s x^{t}\right) Q(x) \\
= & u^{-1}\left(a_{0}+a_{1} x+\cdots+a_{k} x^{k}\right)\left(r^{-1} s x^{t}\right) v^{-1}\left(b_{0}+b_{1} x+\cdots+b_{n} x^{n}\right) u^{-1} \\
= & (u r v)^{-1} p(x)\left(s x^{t}\right) q(x)=0,
\end{aligned}
$$

by using $\sigma^{i}(m)^{-1}=m^{-1}$ for $m \in M$. Thus $M^{-1} R$ is strongly $\bar{\sigma}$-IFP.

The converse can be obtained from that the class of strongly $\sigma$-IFP ring is closed under subrings. 
For $n \geq 2$, denote the $n$ by $n$ full matrix ring over a ring $R$ by $\operatorname{Mat}_{n}(R)$ and the $n$ by $n$ upper triangular matrix ring over $R$ by $U_{n}(R)$.

For a ring $R$ and $n \geq 2$, consider the rings

$$
D_{n}(R)=\left\{\left(\begin{array}{ccccc}
a & a_{12} & a_{13} & \cdots & a_{1 n} \\
0 & a & a_{23} & \cdots & a_{2 n} \\
0 & 0 & a & \cdots & a_{3 n} \\
\vdots & \vdots & \vdots & \ddots & \vdots \\
0 & 0 & 0 & \cdots & a
\end{array}\right) \mid a, a_{i j} \in R\right\}
$$

and

$$
\begin{array}{r}
V_{n}(R)=\left\{m=\left(m_{i j}\right) \in D_{n}(R) \mid m_{s t}=m_{(s+1)(t+1)}\right. \\
\quad \text { for } s=1, \ldots, n-2 \text { and } t=2, \ldots, n-1\},
\end{array}
$$

which are subrings of $\operatorname{Mat}_{n}(R)$. Note that $V_{n}(R) \cong R[x] /\left(x^{n}\right)$, where $\left(x^{n}\right)$ is an ideal of the polynomial ring $R[x]$ over $R$ generated by $x^{n}$.

For a ring $R$ with an endomorphism $\sigma$, the corresponding $\left(a_{i j}\right) \mapsto\left(\sigma\left(a_{i j}\right)\right)$ induces endomorphisms of $\operatorname{Mat}_{n}(R), D_{n}(R)$ and $V_{n}(R)$, respectively. We denote each of them by $\bar{\sigma}$.

Let $A$ be any ring with an endomorphism $\sigma$. Then $\operatorname{Mat}_{n}(A)$ and $U_{n}(A)$ $(n \geq 2)$ do not have IFP since the subring $U_{2}(A)$ is not Abelian. Moreover,

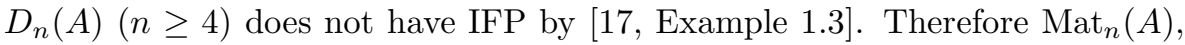
$U_{n}(A)$ for $n \geq 2$ and $D_{n}(A)$ for $n \geq 4$ are not strongly $\bar{\sigma}$-IFP by Proposition 2.4(1). We will freely use this fact without reference. On the other hand, $V_{n}(R)$ $(n \geq 2)$ over a $\sigma$-rigid $\operatorname{ring} R$ is strongly $\bar{\sigma}$-reversible by [15, Theorem 3.1] and so strongly $\bar{\sigma}$-IFP.

But we have the following result.

Proposition 3.7. If $R$ is a $\sigma$-rigid ring, then $D_{n}(R)$ for $n=2,3$ is strongly $\bar{\sigma}-I F P$.

Proof. It is enough to show that $D_{3}(R)$ over a $\sigma$-rigid ring $R$ is strongly $\bar{\sigma}$-IFP since $D_{2}(R)=V_{2}(R)$. Note that $D_{3}(R)[x ; \bar{\sigma}] \cong D_{3}(R[x ; \sigma])$. Suppose that $R$ is $\sigma$-rigid. Let $p(x)=\sum_{i=0}^{m} A_{i} x^{i}, q(x)=\sum_{j=0}^{n} B_{j} x^{j} \in D_{3}(R)[x ; \bar{\sigma}]$ with $A_{i}=\left(a_{s t}^{(i)}\right)$ and $B_{j}=\left(b_{u v}^{(j)}\right) \in D_{3}(R)$. We can write $p(x)=\left(p_{s t}(x)\right), q(x)=$ $\left(q_{u v}(x)\right) \in D_{3}(R[x ; \sigma])$ with $p_{s t}=\sum_{i=0}^{m} a_{s t}^{(i)} x^{i}, q_{u v}=\sum_{j=0}^{n} b_{u v}^{(j)} x^{j} \in R[x ; \sigma]$. From $p(x) q(x)=0$, we have the following equalities:

$$
\begin{gathered}
p_{11}(x) q_{11}(x)=0 ; \\
p_{11}(x) q_{12}(x)+p_{12}(x) q_{11}(x)=0 ; \\
p_{11}(x) q_{13(x)}+p_{12}(x) q_{23}(x)+p_{13}(x) q_{11}(x)=0 ; \\
p_{11}(x) q_{23}(x)+p_{23}(x) q_{11}(x)=0 .
\end{gathered}
$$


Note that $R[x ; \sigma]$ is reduced. Recall that $a b=0$ implies $a A b=0$ and $b a=0$, and that $a b^{2}=0$ implies that $a b=0$ for any reduced $\operatorname{ring} A$ and $a, b \in A$. We will freely use these facts in the following procedure. Since $R$ is strongly $\sigma$-IFP,

$$
p_{11}(x) R[x ; \sigma] q_{11}(x)=0
$$

from Eq. (3). If we multiply Eq. (4) and Eq. (6) on the right-hand side by $q_{11}(x)$ respectively, then we have $p_{12}(x) q_{11}^{2}(x)=0$ and $p_{23}(x) q_{11}^{2}(x)=0$, and so $p_{12}(x) q_{11}(x)=0$ and $p_{23}(x) q_{11}(x)=0$ and moreover, $p_{11}(x) q_{12}(x)=0$ and $p_{11}(x) q_{23}(x)=0$. Thus

$$
\begin{aligned}
& p_{11}(x) R[x ; \sigma] q_{12}(x)=0, p_{12}(x) R[x ; \sigma] q_{11}(x)=0, \\
& p_{11}(x) R[x ; \sigma] q_{23}(x)=0 \text { and } p_{23}(x) R[x ; \sigma] q_{11}(x)=0 .
\end{aligned}
$$

If we multiply Eq. (5) on the right-hand side by $q_{11}(x)$ and $q_{23}(x)$ in turn, then we get $p_{13}(x) q_{11}(x)=0, p_{12}(x) q_{23}(x)=0$ and moreover $p_{11}(x) q_{13(x)}=0$ by the same method as above, respectively. Hence,

$$
p_{11}(x) R[x ; \sigma] q_{13}(x)=0, p_{12}(x) R[x ; \sigma] q_{23}(x)=0 \text { and } p_{13}(x) R[x ; \sigma] q_{11(x)}=0 .
$$

Consequently, Eqs. $(8,9,10)$ imply that $D_{3}(R)$ is strongly $\bar{\sigma}$-IFP.

The hypothesis " $R$ is a $\sigma$-rigid ring" in Proposition 3.7 cannot be weakened the condition " $R$ is a strongly $\sigma$-IFP ring". For example, consider the strongly $\sigma$-IFP ring $R$ which is not $\sigma$-rigid, as in Example 2.11(1). Then $D_{2}(R)$ is not $\sigma$ IFP by [1, Example 2.9], and hence $D_{2}(R)$ is not strongly $\sigma$-IFP by Proposition 2.4(1). Observe that Proposition 3.7 extends the results of $[17$, Propositions 1.2 and 1.6].

For an ideal $I$ and an endomorphism $\sigma$ of a $\operatorname{ring} R$, if $I$ is a $\sigma$-ideal (i.e., $\sigma(I) \subseteq I)$ of $R$ then $\bar{\sigma}: R / I \rightarrow R / I$ defined by $\bar{\sigma}(a+I)=\sigma(a)+I$ for $a \in R$ is an endomorphism of a factor ring $R / I$. The class of strongly $\sigma$-IFP rings is not closed under homomorphic images. Indeed, if $R$ is the ring of quaternions with integer coefficients with a monomorphism $\sigma$, then $R$ is a domain, and so strongly $\sigma$-IFP; while for any odd prime integer $q$, we have $R / q R \cong \operatorname{Mat}_{2}\left(\mathbb{Z}_{q}\right)$ by the argument in $[6$, Exercise $2 \mathrm{~A}]$. But $\operatorname{Mat}_{2}\left(\mathbb{Z}_{q}\right)$ is not strongly $\bar{\sigma}$-IFP as mentioned earlier, and thus the factor ring $R / q R$ is not strongly $\bar{\sigma}$-IFP.

For a nonempty subset $S$ of a ring $R$, we write $r_{R}(S)=\{c \in R \mid S c=$ $0\}$ (resp., $\ell_{R}(S)=\{c \in R \mid c S=0\}$ ) which is called the right (resp., left) annihilator of $S$ in $R$. A ring $R$ has IFP if and only if any one-sided annihilator is an ideal of $R$ by [26, Lemma 1.2].

Proposition 3.8. (1) Let $R$ be a strongly $\sigma-I F P$ ring. If $I$ is the one-sided annihilator of any nonempty subset in $R$ and $\sigma(I) \subseteq I$, then $R / I$ is strongly $\bar{\sigma}-I F P$.

(2) Let $\sigma$ be an endomorphism and $I$ an ideal of a ring $R$. For a $\sigma$-ideal $I$ of $R$ such that $R / I$ is strongly $\bar{\sigma}-I F P$, if $I$ is a $\sigma$-rigid ring without identity, then $R$ is strongly $\sigma-I F P$. 
Proof. (1) Assume that $R$ is a strongly $\sigma$-IFP ring and let $I=r_{R}(S)$ for $\phi \neq S \subseteq R$. Then $I$ is an ideal since $R$ has IFP by Proposition 2.4(1). Let $\bar{p}(x) \bar{q}(x)=\overline{0}$ with $\bar{p}(x)=\sum_{i=0}^{m} \bar{a}_{i} x^{i}, \bar{q}(x)=\sum_{j=0}^{n} \bar{b}_{j} x^{j} \in \bar{R}[x ; \bar{\sigma}]$ where $\bar{R}=$ $R / I$ and $\bar{c}=c+I$ for $c \in R$. Then $S p(x) q(x)=0$, and so $S p(x)\left(r x^{t}\right) q(x)=0$ for any $r \in R$ and $t \geq 0$ by hypothesis, where $p(x)=\sum_{i=0}^{m} a_{i} x^{i}, q(x)=$ $\sum_{j=0}^{n} b_{j} x^{j} \in R[x ; \sigma]$. Thus

$$
S\left(\sum_{k=0}^{m+n}\left(\sum_{i+j=k} a_{i} \sigma^{i}\left(r \sigma^{t}\left(b_{j}\right)\right)\right) x^{k+t}\right)=0
$$

and so

$$
\sum_{k=0}^{m+n}\left(\sum_{i+j=k} \bar{a}_{i} \bar{\sigma}^{i}\left(\bar{r} \bar{\sigma}^{t}\left(\bar{b}_{j}\right)\right)\right) x^{k+t}=\overline{0},
$$

entailing that $\bar{p}(x)\left(\bar{r} x^{t}\right) \bar{q}(x)=\overline{0}$ for any $r \in R$ and $t \geq 0$. Therefore $R / I$ is strongly $\bar{\sigma}$-IFP. The left annihilator case is similar.

(2) Assume that $R / I$ is strongly $\bar{\sigma}$-IFP and $I$ is a $\sigma$-rigid ring. Let $p(x) q(x)=$ 0 for $p(x), q(x) \in R[x ; \sigma]$. Then $p(x) R[x ; \sigma] q(x) \subseteq I[x ; \sigma]$ since $R / I$ is strongly $\bar{\sigma}$-IFP, and $q(x) I[x ; \sigma] p(x)=0$ since $q(x) I[x ; \sigma] p(x) \subseteq I[x ; \sigma],(q(x) I[x ; \sigma] p(x))^{2}$ $=0$ and $I[x ; \sigma]$ is reduced. Thus, $(p(x) R[x ; \sigma] q(x) I[x ; \sigma])^{2}=p(x) R[x ; \sigma] q(x)$ $I[x ; \sigma] p(x) R[x ; \sigma] q(x) I=0$ and so $p(x) R[x ; \sigma] q(x) I[x ; \sigma]=0$; hence

$$
(p(x) R[x ; \sigma] q(x))^{2} \subseteq p(x) R[x ; \sigma] q(x) I[x ; \sigma]=0
$$

since $p(x) R[x ; \sigma] q(x) \subseteq I[x ; \sigma]$. Then $p(x) R[x ; \sigma] q(x)=0$ since $I[x ; \sigma]$ is reduced. Therefore $R$ is strongly $\sigma$-IFP.

The condition " $I$ is a $\sigma$-rigid ring without identity" in the Proposition 3.8(2) cannot be dropped by the next example.

Example 3.9. Consider a ring $R=U_{2}(F)$ over a division $\operatorname{ring} F$ and an automorphism $\sigma$ of $R$ defined by $\sigma\left(\left(\begin{array}{ll}a & b \\ 0 & c\end{array}\right)\right)=\left(\begin{array}{ll}a & -b \\ 0 & c\end{array}\right)$. Then $R$ is not clearly Abelian, and so $R$ is not strongly $\sigma$-IFP by Corollary 2.5(1). The $\sigma$-ideal $I=\left(\begin{array}{cc}F & F \\ 0 & 0\end{array}\right)$ of $R$ is not $\sigma$-rigid as a ring without identity: Indeed, $A \sigma(A)=0$ but $A \neq 0$ for $A=\left(\begin{array}{ll}0 & 1 \\ 0 & 0\end{array}\right) \in I$. Note that the factor $\operatorname{ring} R / I \cong F$ is reduced and $\bar{\sigma}$ is an identity map on $R / I$. That is, $R / I$ is $\bar{\sigma}$-rigid and hence strongly $\bar{\sigma}$-IFP.

Acknowledgments. The authors thank the referee for very careful reading of the manuscript and many valuable suggestions that improved the paper by much.

\section{References}

[1] M. Başer, A. Harmanci, and T. K. Kwak, Generalized semicommutative rings and their extensions, Bull. Korean Math. Soc. 45 (2008), no. 2, 285-297.

[2] M. Başer, T. K. Kwak, and Y. Lee, The McCoy condition on skew polynomial rings, Comm. Algebra 37 (2009), no. 11, 4026-4037. 
[3] H. E. Bell, Near-rings in which each element is a power of itself, Bull. Austral. Math. Soc. 2 (1970), 363-368.

[4] P. M. Cohn, Reversible rings, Bull. London Math. Soc. 31 (1999), no. 6, 641-648.

[5] J. L. Dorroh, Concerning adjunctins to algebras, Bull. Amer. Math. Soc. 38 (1932), $85-88$.

[6] K. R. Goodearl and R. B. Warfield, Jr., An Introduction to Noncommutative Noetherian Rings, Cambridge University Press, 1989.

[7] Y. Hirano, On the uniqueness of rings of coefficients in skew polynomial rings, Publ. Math. Debrecen 54 (1999), no. 3-4, 489-495.

[8] C. Y. Hong, N. K. Kim, and T. K. Kwak, Ore extensions of Baer and p.p.-rings, J. Pure Appl. Algebra. 151 (2000), no. 3, 215-226.

[9] - On skew Armendariz rings, Comm. Algebra 31 (2003), no. 1, 103-122.

[10] C. Y. Hong, N. K. Kim, and Y. Lee, Skew polynomial rings over semiprime rings, J. Korean Math. Soc. 47 (2010), no. 5, 879-897.

[11] Extensions of McCoy's theorem, Glasgow Math. J. 52 (2010), no. 1, 155-159.

[12] C. Y. Hong, T. K. Kwak, and S. T. Rizvi, Extensions of generalized Armendariz rings, Algebra Colloq. 13 (2006), no. 2, 253-266.

[13] C. Huh, Y. Lee, and A. Smoktunowicz, Armendariz rings and semicommutative rings, Comm. Algebra 30 (2002), no. 2, 751-761.

[14] D. A. Jordan, Bijective extensions of injective rings endomorphism, J. London Math. Soc. 25 (1982), no. 3, 435-448.

[15] F. Kaynarca, T. K. Kwak, and Y. Lee, Reversibility of skew polynomial rings (submitted).

[16] N. K. Kim, T. K. Kwak, and Y. Lee, Insertion-of-factors-property skewed by ring endomorphisms, Taiwanese J. Math. 18 (2014), no. 3, 849-869.

[17] N. K. Kim and Y. Lee, Extensions of reversible rings, J. Pure Appl. Algebra 185 (2003), no. 1-3, 207-223.

[18] J. Krempa, Some examples of reduced rings, Algebra Colloq. 3 (1996), no. 4, 289-300.

[19] T. K. Kwak, Y. Lee, and S. J. Yun, The Armendariz property on ideals, J. Algebra 354 (2012), 121-135.

[20] J. Lambek, Lectures on Rings and Modules, Blaisdell Publishing Company, Waltham, 1966.

[21] N. H. McCoy, Remarks on divisors of zero, Amer. Math. Monthly 49 (1942), 286-295.

[22] L. Motais de Narbonne, Anneaux semi-commutatifs et unis riels anneaux dont les id aux principaux sont idempotents, Proceedings of the 106th National Congress of Learned Societies (Perpignan, 1981), 71-73, Bib. Nat., Paris, 1982.

[23] A. R. Nasr-Isfahani and A. Moussavi, Skew Laurent polynomial extensions of Baer and p.p.-rings, Bull. Korean Math. Soc. 46 (2009), no. 6, 1041-1050.

[24] P. P. Nielsen, Semi-commutativity and the McCoy condition, J. Algebra 298 (2006), $134-141$.

[25] M. B. Rege and S. Chhawchharia, Armendariz rings, Proc. Japan Acad. Ser. A Math. Sci. 73 (1997), no. 1, 14-17.

[26] G. Shin, Prime ideals and sheaf representation of a pseudo symmetric ring, Trans. Amer. Math. Soc. 184 (1973), 43-60.

Muhittin BaşER

Department of Mathematics

KocATEPE UNIVERSITY

AFyonkarahisar 03200, TURKEy

E-mail address: mbaser@aku.edu.tr 
BEGÜM HiÇYiLmaZ

Department of Mathematics

KOCATEPE UNIVERSITY

Afyonkarahisar 03200, Turkey

E-mail address: bgmhcylmz@hotmail.com

FATMA Kaynarca

Department of Mathematics

Kocatepe University

Afyonkarahisar 03200, Turkey

E-mail address: fkaynarca@aku.edu.tr

TAi Keun KWAK

Department of Mathematics

DAEJIN UNIVERSITY

Pocheon 487-711, Korea

E-mail address: tkkwak@daejin.ac.kr

YANG LEE

Department of Mathematics

Pusan National University

PUSAN 609-735, KoreA

E-mail address: ylee@pusan.ac.kr 